\title{
Electron Beam Induced Current Characterization of Dark Line Defects in Failed and Degraded High Power Quantum Well Laser Diodes
}

\author{
Maribeth Mason, Nathan Presser, Yongkun Sin, Brendan Foran, and Steven C. Moss* \\ * Electronics and Photonics Laboratory, The Aerospace Corporation, El Segundo, CA 90405
}

Multimode InGaAs/AlGaAs strained quantum well single emitter laser diodes at 920-980 nm have become indispensable as pump lasers for various applications. Most reports have focused on performance characteristics, with limited reports on understanding the physics of failure or reliability. In this paper, we investigate the dependence of electron beam induced current (EBIC) contrast from dark-line defects (DLDs) in failed and degraded high power quantum well laser diodes (HPLDs) on temperature and voltage bias. These results can be correlated to destructive physical analysis (DPA) and other defect characterization techniques to provide insight into failure mechanisms in these devices. Voltage bias induced contrast variations in EBIC allowed us to make the first observation of what may be the DLD initiation point in a degraded, but not failed, HPLD.

The EBIC capability is integrated with a dual-beam system combining a scanning electron microscope (SEM) with a focused ion beam (FIB). Defects not visible with SEM can be observed with EBIC, as shown in Figure 1, and targeted for DPA using the FIB [1]. Defect depth profiles are obtained by varying the electron accelerating voltage, or applying an external bias to modulate the depletion width. Other defect properties can be extracted from temperature-dependent EBIC contrast. By correlating temperature and voltage dependent EBIC to DPA and other techniques, we hope to build a database of defect types in failed and degraded HPLDs.

Figure 1 shows the temperature and voltage bias dependent contrast for a passivated HPLD with a $10 \%$ loss in output power. The dark spot near the rear facet of the HPLD may correspond to a DLD initiation point. Figure 2 shows that, at $25 \mathrm{C}$ and $0 \mathrm{~V}$, a dark spot in the EBIC contrast is visible. At reverse bias, an extended defect structure is observed surrounding the central spot. Under reverse bias, the EBIC contrast of this region increases due to expansion of the depletion width of the $p-n$ junction, but the contrast changes little under forward bias. The EBIC contrast for defects far from the central spot is also enhanced at high temperature due to a decrease in the minority carrier diffusion length. Based on prior observations [2], the central spot may correspond to an area where the quantum well is severely disturbed, while the surrounding region may possess a high defect density without a quantum well disturbance. Further DPA is planned to confirm this hypothesis.

Figure 3 summarizes the temperature and voltage dependent contrast data for two pixels located in the center spot and outer spot regions, respectively, of the HPLD. These two pixels are observed to exhibit different temperature and voltage dependent contrast. The EBIC experiments produce a fivedimensional data set, where each $\mathrm{x}$ and $\mathrm{y}$ coordinate of the EBIC image has a contrast associated with it that varies with temperature and voltage. We will discuss algorithms based on wavelet transforms to simplify the data interpretation, and correlate the results to DPA and other defect characterization techniques.

[1] N. Presser et al., Microsc. Microanal. 14 (Suppl. 2) (2008) 392-393

[2] B. Foran et al., Proc. IEEE CLEO/QELS 2009, 1-2. 
[3] This work was supported under The Aerospace Corporation's Independent Research and Development Program.
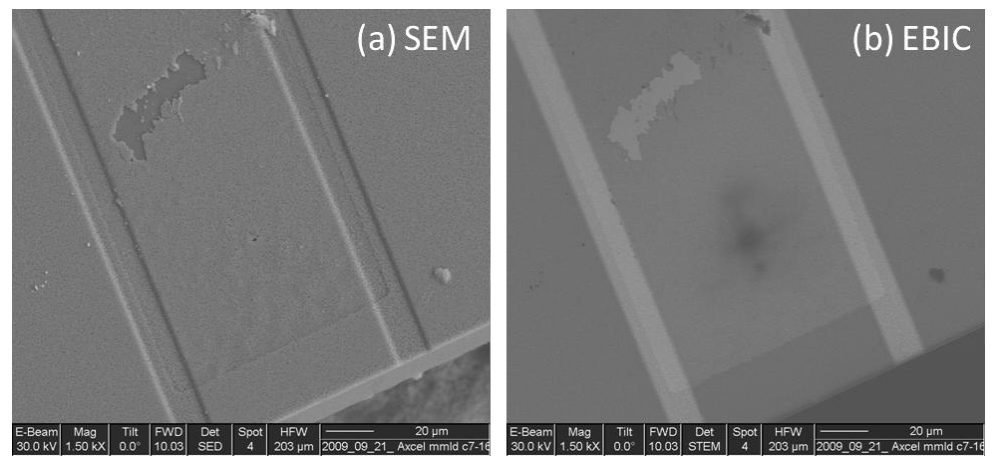

Figure 1. (a) SEM and (b) EBIC images of possible DLD initiation point in $10 \%$ degraded HPLD at $0 \mathrm{~V}$ bias and $298 \mathrm{~K} .$.
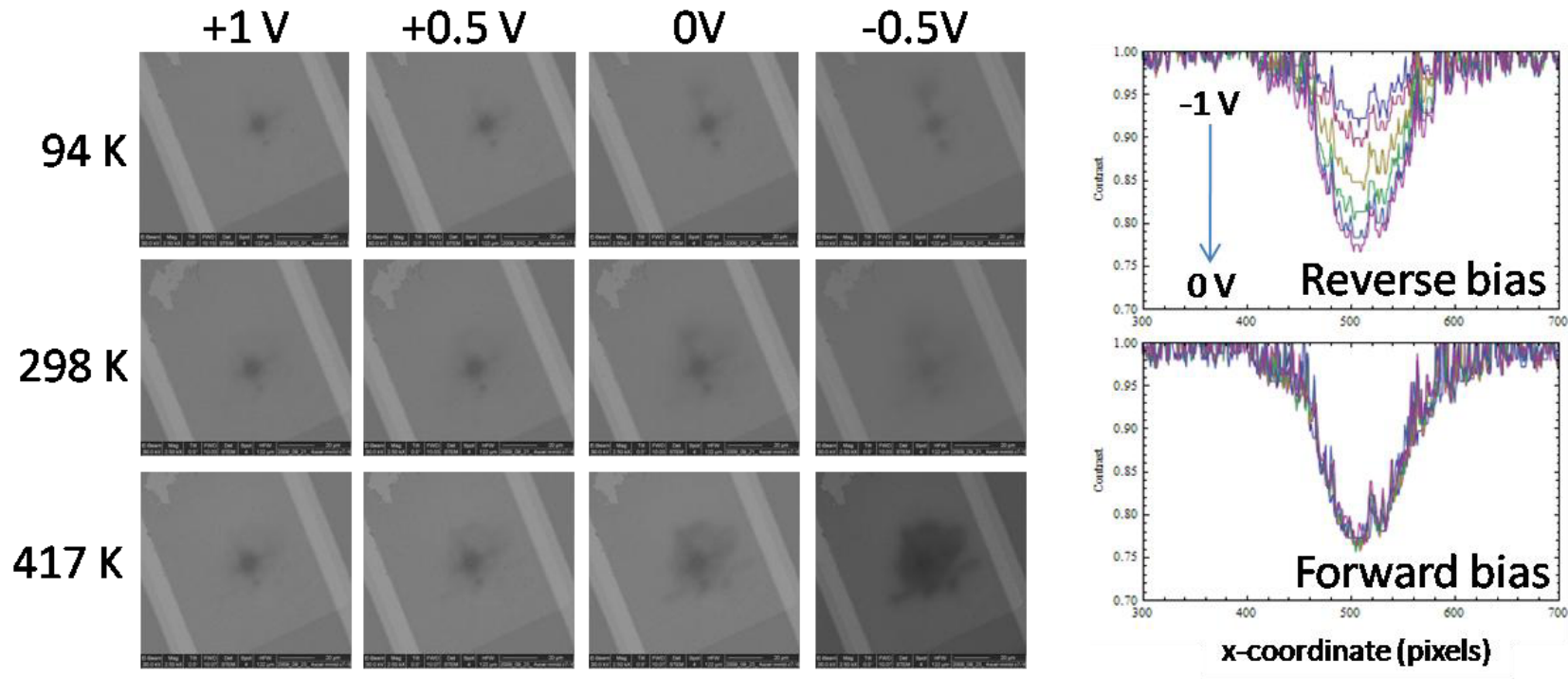

Figure 2. Temperature and voltage dependent EBIC contrast of possible DLD initiation point in $10 \%$ degraded HPLD. Voltage-dependent EBIC contrast data (graphs at right) is given for 94K.
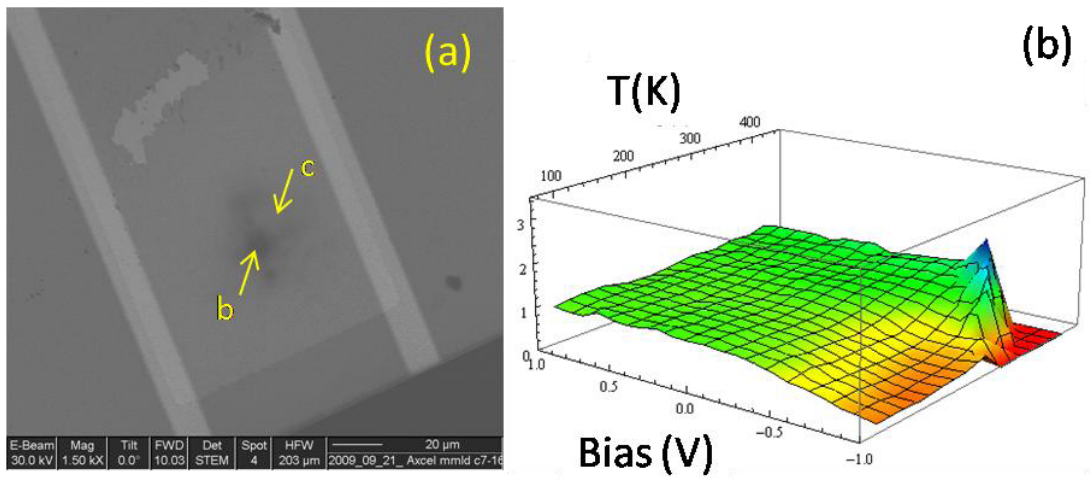

(b)

(c)

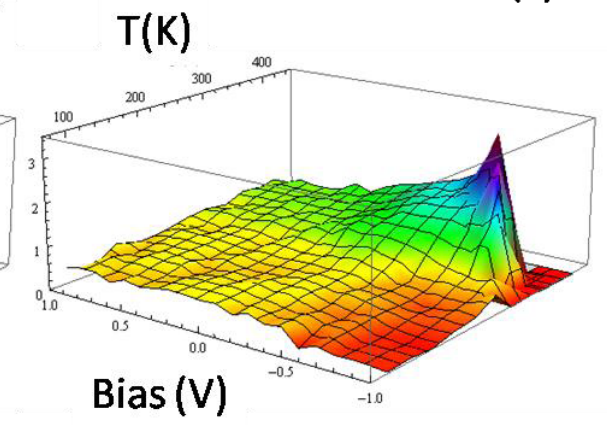

Figure 3. (a) EBIC image of HPLD illustrating regions for temperature and voltage dependent contrast analysis. (b) Temperature and voltage dependent contrast of center defect region labeled in (a). (c) Temperature and voltage dependent contrast of outer defect region labeled in (a). 